\title{
A Health Department's Collaborative Model for Disease Surveillance Capacity Building
}

\author{
Ikechi Konkwo*1,2, Robert G. Harmon ${ }^{1}$, William C. Livingood ${ }^{2,1}$, Thomas BryantIII' and \\ Saad Zaheer ${ }^{1}$
}

${ }^{1}$ Institute for Public Health Informatics and Research, Duval County Health Dept, Jacksonville, FL, USA; ${ }^{2}$ Center for Health Equity and Research - University of Florida/Shands Medical Center, Jacksonville, FL, USA

\section{Objective}

Highlight one academic health department's unique approach to optimizing collaborative opportunities for capacity development and document the implications for chronic disease surveillance and population health.

\section{Introduction}

Public Health departments are increasingly called upon to be innovative in quality service delivery under a dwindling resource climate as highlighted in several publications of the Institute of Medicine. Collaboration with other entities in the delivery of core public health services has emerged as a recurring theme. One model of this collaboration is an academic health department: a formal affiliation between a health professions school and a local health department. Initially targeted at workforce development, this model of collaboration has since yielded dividends in other core public health service areas including community assessment, program evaluation, community-based participatory research and data analysis.

The Duval County Health Department (DCHD), Florida, presents a unique community-centered model of the academic health department. Prominence in local informatics infrastructure capacity building and hosting a CDC-CSTE applied public health informatics fellowship (APHIF) in the Institute for Public Health Informatics and Research (IPHIR) in partnership with the Center for Health Equity Research, University of Florida \& Shands medical center are direct dividends of this collaborative model.

\section{Methods}

We examined the collaborative efforts of the DCHD and present the unique advantages these have brought in the areas of entrenched data-driven public health service culture, community assessments, program evaluation, community-based participatory research and health informatics projects.

\section{Results}

Advantages of the model include a data-driven culture with the balanced scorecard model in leadership and sub-departmental emphases on quality assurance in public health services. Activities in IPHIR include data-driven approaches to program planning and grant developments, program evaluations, data analyses and impact assessments for the DCHD and other community health stakeholders.

Reports developed by IPHIR have impacted policy formulation by highlighting the need for sub county level data differentiation to address health disparities. Unique community-based mapping of Duval County into health zones based on health risk factors correlating with health outcome measures have been published. Other reports highlight chronic disease surveillance data and health scorecards in special populations.
Partnerships with regional higher institutions (University of Florida, University of North Florida and Florida A\&M University) increased public health service delivery and yielded rich communitybased participatory research opportunities.

Cutting edge participation in health IT policy implementation led to the hosting of the fledgling community HIE, the Jacksonville Health Information Network, as well as leadership in shaping the landscape of the state HIE. This has immense implications for public health surveillance activities as chronic disease surveillance and public health service research take center stage under new healthcare payment models amidst increasing calls for quality assurance in public health services.

DCHD is currently hosting a CDC-funded fellowship in applied public health informatics. Some of the projects materializing from the fellowship are the mapping of the current public health informatics profile of the DCHD, a community based diabetes disease registry to aid population-based management and surveillance of diabetes, development of a proposal for a combined primary care/general preventive medicine residency in UF-Shands Medical Center, Jacksonville and mobilization of DCHD healthcare providers for the roll-out of the state-built electronic medical records system (Florida HMS-EHR).

\section{Conclusions}

Academic health centers provide a model of collaboration that directly impacts on their success in delivering core public health services. Disease surveillance is positively affected by the diverse community affiliations of an academic health department. The academic health department, as epitomized by DCHD, is also better positioned to seize up-coming opportunities for local public health capacity building.

\section{Keywords}

Academic Health Departments; collaborative model; health informatics projects

\section{Acknowledgments}

This study was supported in part by an appointment to the APHIF Program administered by CSTE and funded by the CDC Cooperative Agreement 3U38HM000414-04W1.

\section{*Ikechi Konkwo}

E-mail: Ikechi_Konkwo@doh.state.fl.us 